\title{
Cherenkov radiation of a charge in axicon-based dielectric concentrator
}

\author{
Sergey N. Galyamin $\odot^{*}$ and Andrey V. Tyukhtin \\ Saint Petersburg State University, 7/9 Universitetskaya nab., St. Petersburg, 199034 Russia
}

(Received 28 August 2020; accepted 26 October 2020; published 13 November 2020)

\begin{abstract}
We propose a new type of axisymmetric dielectric target which effectively concentrates Cherenkov radiation (CR) generated in the bulk of the material into a small vicinity of a focus point. It can be called the "axicon-based concentrator for CR." A theoretical investigation of radiation field produced by a charge moving through the discussed radiator is performed for the general case where a charge trajectory is shifted with respect to the structure axis. The idea of a dielectric target with a specific profile of the outer surface was presented and developed in our preceding papers. However, contrary to the previous configuration of such a target (which was investigated for both centered and shifted charge trajectory), the current version of the device allows the efficient concentration of CR energy from relativistic particles, making this device extremely prospective for various applications.
\end{abstract}

DOI: 10.1103/PhysRevAccelBeams.23.113001

\section{INTRODUCTION}

Electromagnetic radiation emerging during an interaction between charged particle beams and various structures (homogeneous media, periodic structures etc.) were widely used for decades in various applications. For example, ordinary vacuum devices (klystrons, backward-wave oscillators, gyrotrons) and outstanding in power and spatiotemporal resolution of the pulse $\mathrm{x}$-ray free-electron lasers are both based on beam interaction with periodic "structure" (be it a real structure or a specially structured external field) resulting in appropriate beam transformation and essential gain of radiation intensity. When the medium with which the beam is interacted is homogeneous Cherenkov radiation (CR) occurs even in the case of uniform motion with a velocity exceeding light speed in the given medium [1-5]. For years, CR was intensively studied in various contexts, while the most recent between them are dielectric wakefield acceleration [6-8], bunch size measurement [911 , and contemporary sources of radiation, including those for terahertz $(\mathrm{THz})$ frequencies [12-18] (note that Cherenkov-type radiation from optically rectified laser pulses $[19,20]$ is also considered as a convenient way to produce $\mathrm{THz}$ radiation in dielectric-based converters $[21,22])$. It is worth noting that the idea of using the CR effect for producing the radiation is not new, but in recent years it has been essentially improved. If a bunch used for generation is already of proper quality, i.e., has a

\footnotetext{
*s.galyamin@spbu.ru
}

Published by the American Physical Society under the terms of the Creative Commons Attribution 4.0 International license. Further distribution of this work must maintain attribution to the author(s) and the published article's title, journal citation, and DOI. considerable charge and low emittance, is essentially short and small in transverse directions (this is typically so for relativistic bunches produced by modern accelerators) then $\mathrm{GeV}$-per-meter CR fields can be potentially obtained in relatively simple dielectric-loaded structures [8]. Noninvasive bunch diagnostics based on prolonged dielectric targets of complicated shape is another modern area for CR applications [9-11] which possess several advantages compared to traditional schemes based on transition or diffraction radiation.

The last-mentioned area involves the need to calculate CR produced by dielectric target with several boundaries and edges which is marginally possible to do rigorously. To resolve this issue with reliable accuracy we have been developing for several recent years an original combined approach based on certain "etalon" problem, ray-optics laws and Stratton-Chu formulas (see, for example, [23-26]). It is worth noting that this approach has been approved by direct comparison between its results and results of numerical simulations in COMSOL Multiphysics [27].

Several papers from this list closely relate to the present paper because they dealt with the axisymmetric dielectric target- "dielectric concentrator for CR"-focusing the majority of generated CR in a small vicinity of a predetermined point (focus) without any additional lenses or mirrors [23,25-27]. While possibilities of this concentrator for radiation intensity enhancement, beam position, and velocity measurements are rather attractive, an essential disadvantage is that this target has more or less convenient dimensions for relatively slow charged particles only. Therefore it would be of considerable practical importance to eliminate the mentioned shortcoming and allow the concentration of $\mathrm{CR}$ produced by a relativistic charged particle. The solution to this problem is the main goal of the present paper. 
We propose here an "axicon-based concentrator for CR"- - a new type of axisymmetric dielectric target which concentrates the main portion of CR into a focus point. Contrary to the previous "single-refraction" configuration [23], this target uses one reflection and one refraction of CR rays. For reflection, it is convenient to use a hollow conical target (axicon) in the geometry investigated separately in a recent paper [28]. It is important here that the cone apex angle can be adjusted so that CR rays will form a paraxial beam with respect to the charge trajectory after the reflection for arbitrary angle of incidence (i.e., for arbitrary charge velocity). Therefore the discussed concentrator can be designed for effective focusing of CR from charged particle bunches with arbitrary velocity including relativistic velocities which are of most practical interest. Moreover, we investigate here the effect of charge shift from the symmetry axis, similarly to the analogous investigation for "single-refraction" concentrator $[25,26]$.

It should be especially noted that polarization of CR in the discussed concentrator is nearly radial in the region close to the output surface (inside the "lens") resulting in nearly longitudinal polarization of CR field in the vicinity of the symmetry axis within the focal spot. In turn, radially polarized laser beams are extensively studied in recent years due to their unique properties (for example, a smaller focal spot size compared to linearly or circularly polarized light, including even subdiffraction focal spot) [29-31] and prospective applications, including laser particle acceleration in vacuum [32-34]. In this context, the discussed "axicon-based concentrator for CR" becomes even more prospective since it allows the implementation of mentioned attractive phenomena to $\mathrm{CR}$ from relativistic bunches produced by modern accelerators.

\section{PROBLEM FORMULATION}

Figure 1 shows the geometry of the problem under investigation. Note that along with the Cartesian frame $(x, y, z)$, a corresponding cylindrical frame $(\rho, \varphi, z)$ is introduced. A point charge $q$ moves with a constant velocity $v=\beta c$ along a straight trajectory inside the channel in the axisymmetric dielectric target with permittivity $\varepsilon$ and permeability $\mu=1$ (for convenience). It is supposed here that condition for CR generation is fulfilled, i.e., $\sqrt{\varepsilon} \beta>1$. The position of charge trajectory in $x y$-plane is determined by $r_{0}$ and $\varphi_{0}$, see Fig. 1(b).

The target consists of two "glued" bodies of revolution: a hollow axicon (see, for example, [35] for the description of this optical element) and a hollow "lens." The cone is determined by its apex angle $\alpha$ while cylindrical coordinates $\rho=\rho_{0}, z=z_{0}$ of the outer profile of the lens are determined as follows (note that this profile can be deduced using the same considerations as in $[23,25])$ :

$$
\begin{aligned}
\rho_{0}(u) & =r(u) \sin (u), \\
z_{0}(u) & =z_{f}-r(u) \cos (u),
\end{aligned}
$$

where

$$
r(u)=f(\sqrt{\varepsilon}-1)[\sqrt{\varepsilon} \cos (u)-1]^{-1},
$$

$f$ is a "focal" parameter. The maximum transverse size of the target $x_{\max }$ determines the maximum angle $u_{\max }$, the minimum angle $u_{\min }$ is determined by the channel radius $a$. Total length of the target including the imaginary "nose" of the cone is $z_{f}-f$, where $z_{f}=x_{\max }\left(\cot \alpha+\cot u_{\max }\right)$. It should be underlined that the surface (2) is typically
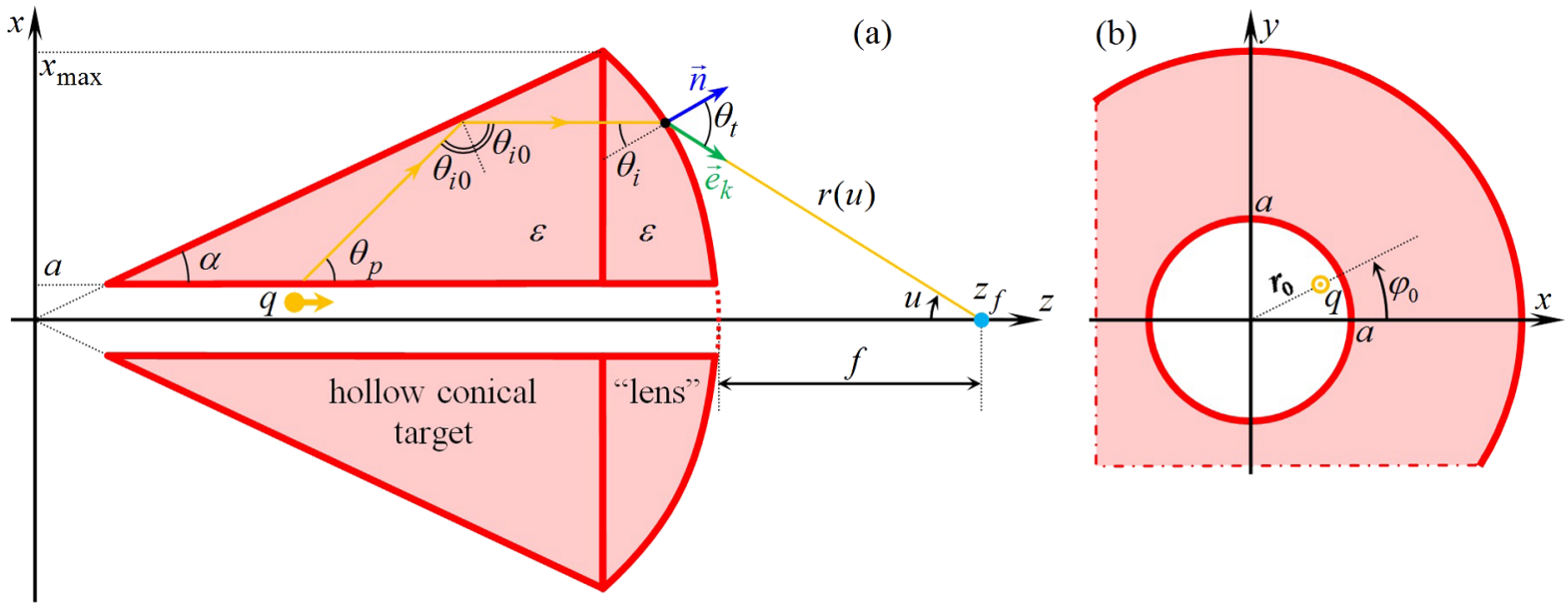

FIG. 1. Geometry of the problem and main notations. (a) $(z x)$-cut of the "axicon-based concentrator for CR": a hollow "lens" is attached to the output surface of a hollow conical target having its apex facing the incident charged particle bunch. Both revolution bodies are made from the same dielectric material with permittivity $\varepsilon$. The outer profile of the "lens" is hyperbolic and is determined by the function $r(u)$. A point charge $q$ moves along the straight trajectory shifted with respect to the $z$-axis. Depicted parameters are discussed in the text. (b) ( $x y)$-cut of the target (the channel radius is enlarged for convenience) and the position of the charge shifted trajectory. 
designed so that a beam of rays parallel to $z$-axis converges to exact focus $z=z_{f}$ after the refraction. Schematic propagation of one such ray through the target to the focus is shown in Fig. 1. It is of essential importance that for "axicon-based concentrator for CR" we have two independent parameters for design, charge velocity $\beta$ and cone angle $\alpha$. This means, for example, that we can adjust the cone angle $\alpha$ so that CR rays will be parallel to $z$-axis for arbitrary charge velocity including relativistic velocities which are of most practical importance. Moreover, if CR rays are parallel to $z$-axis then $C R$ has radial polarization and results in the longitudinally polarized field after focusing. These points will be discussed again below.

Our further analysis is at first based on the analytical solution of the corresponding "etalon" problem allowing determination of initial CR rays inside the bulk of dielectric. In short, this problem takes into account the interaction of a moving charge with the target's boundary closest to its trajectory while all other boundaries are neglected. In the case under consideration, we should solve for Maxwell equations in an infinite dielectric with a vacuum channel inside which a point charge propagates along the shifted trajectory. Corresponding details can be found in the main text and Appendix B of Ref. [25] while small corrections are given in [26].

Important note to this solution (which is an infinite sum of "harmonics") is the following: the phase of all summands can be written as

$$
\exp \left[i k_{0} \beta^{-1}\left(z+\sqrt{\varepsilon \beta^{2}-1} \rho\right)\right]
$$

i.e., in the same manner as in the symmetric case $r_{0}=0$ (here $k_{0}=\omega / c, \omega$ is a frequency, $c$ is a light speed in vacuum). Therefore all results concerning the structure of $\mathrm{CR}$ rays inside the hollow conical target for the symmetric case (see [28]) are fully applicable to the present problem. In particular, we should consider only the "main" wave of two analyzed in [28] because the lens surface (2) has been designed for the concentration of paraxial CR rays which correspond to this wave (it is worth noting that in the case of flat output surface it is this wave which is responsible for "Cherenkov spotlight" effect-an effect of essential CR enhancement in the $z$-direction in the far-field area due to the constructive interference, see [28] for details). In our case, propagation of this wave is the same until ray refraction at the output surface because now the lens is attached there. This last refraction can be analyzed similarly to the case of a "single-refraction" concentrator. It should be also noted that in this paper we neglect absorption in dielectric for simplicity consideration. However, small dissipation can be easily incorporated in both solutions of the etalon problem and ray-optics derivations in hollow axicon. For example, real material (Teflon) absorption has been taken into account in calculations of CR from a "single-refraction" concentrator in [23].

\section{STRATTON-CHU FORMALISM}

According to our method, we utilize the Stratton-Chu formulas $[36,37]$ to calculate CR exiting the target. Recall that these integral formulas give exact result if tangential electric and magnetic fields are determined exactly at the surface of the integration (the aperture $S_{a}$ ). In this paper, we use the form of these formulas from [37] (see also our papers $[24,25,38,39])$ with the outer surface of the target (1) as the aperture:

$$
\begin{aligned}
4 \pi \vec{E}_{\omega}= & \int_{S_{a}}\left\{i k_{0}\left[\vec{n}, \vec{H}_{\omega}^{a}\right] \psi+\frac{i}{k_{0}}\left(\left[\vec{n}, \vec{H}_{\omega}^{a}\right], \vec{\nabla}\right) \vec{\nabla} \psi\right. \\
& \left.+\left[\left[\vec{E}_{\omega}^{a}, \vec{n}\right], \vec{\nabla} \psi\right]\right\} d \Sigma,
\end{aligned}
$$

where $\psi$ is a Green function,

$$
\begin{aligned}
& \psi=\exp \left(i k_{0} \tilde{R}\right) / \tilde{R}, \\
& \tilde{R}=\sqrt{\left(x-x_{0}\right)^{2}+\left(y-y_{0}\right)^{2}+\left(z-z_{0}\right)^{2}},
\end{aligned}
$$

$d \Sigma$ is a surface element of $S_{a}$. As follows from Eq. (4), electromagnetic (EM) field outside the target is determined by the tangential electric and magnetic fields at the aperture $S_{a}$. We utilize the following parametrization of the Cartesian coordinates of the aperture via angles $u$ and $\varphi$ :

$x_{0}(u, \varphi)=\rho_{0}(u) \cos \varphi, \quad y_{0}(u, \varphi)=\rho_{0}(u) \sin \varphi$,

while $z_{0}(u, \varphi)$ is given by (2) together with $\rho_{0}(u)$. In order to calculate the parameters of the surface, it is convenient to use the tensor formalism by V.A. Fock $[38,40]$ and determine the metric tensor of the surface $g$. Thus, for the elementary square of the surface we obtain $d \Sigma=\sqrt{g(u)} d u d \varphi$, where

$$
\sqrt{g(u)}=\frac{r^{2}(u) \sin u \sqrt{1-2 \sqrt{\varepsilon} \cos u+\varepsilon}}{\sqrt{\varepsilon} \cos u-1} .
$$

The components of the external unit normal $\vec{n}$ are

$$
\begin{aligned}
& n_{\rho}=\frac{\sin u}{\sqrt{1-2 \sqrt{\varepsilon} \cos u+\varepsilon}}, \\
& n_{z}=\frac{\sqrt{\varepsilon}-\cos u}{\sqrt{1-2 \sqrt{\varepsilon} \cos u+\varepsilon}} .
\end{aligned}
$$

In order to find the fields $\vec{E}_{\omega}^{a}$ and $\vec{H}_{\omega}^{a}$, we use the same approved method as in papers [25,27]. Moreover, the corresponding etalon problem is the same as in [25]. The solution of the etalon problem should be 
complemented by the analysis of the "main" CR wave reflection at the cone generatrix, this has been done in [28]. In particular, the way of this ray is the following, see Fig. 1. It incidents the boundary formed by cone generatrix at the angle

$$
\theta_{i 0}=\pi / 2+\alpha-\theta_{p},
$$

where

$$
\theta_{p}=\arccos \left[\frac{1}{\sqrt{\varepsilon} \beta}\right]
$$

is a Cherenkov angle (it reduces to $\cos \theta_{p}=1 / \sqrt{\varepsilon}$ for $\beta \rightarrow 1$ ), reflected at the same angle and some portion of it is refracted at the angle

$$
\theta_{t 0}=\arcsin \left(\sqrt{\varepsilon} \sin \theta_{i 0}\right)
$$

with respect to the generatrix normal. Then the reflected wave propagates to the cone base and passes through the cone base flat surface at the angle

$$
\theta_{i}^{\prime}=2 \alpha-\theta_{p}
$$

with respect to $z$-axis. When $\theta_{i}^{\prime}=0$ the rays form a parallel beam before the lens, this is the case where all the rays converge exactly to the focus point. This is a very important feature of the discussed "axicon-based concentrator" that we have two parameters to obtain the parallel beam of rays: $\alpha$ and $\beta$. Most convenient way is to adjust $\alpha$ in accordance with the "designed" charged particle velocity $\beta_{0}$, i.e., to choose

$$
\alpha=\frac{1}{2} \theta_{p 0}=\frac{1}{2} \arccos \left(\frac{1}{\sqrt{\varepsilon} \beta_{0}}\right),
$$

which means that cone angle is a half the Cherenkov angle $\theta_{p 0}$ and that CR rays for $\beta=\beta_{0}$ propagate parallel to $z$-axis after reflection from the axicon generatrix. Also this possibility is the most distinguishing point compared to the single-refraction concentrator $[23,25,26]$ which can be designed for single charge velocity only.

Due to the asymmetry caused by charge shift, CR of both polarizations is generated. "Parallel" polarization $(\|)$ contains components $E_{z \omega}, E_{\rho \omega}$, and $H_{\varphi \omega}$. The corresponding Fresnel reflection coefficient (responsible for the reflection at the cone generatrix) is

$$
R_{\|}=\frac{\cos \theta_{i 0}-\sqrt{\varepsilon} \cos \theta_{t 0}}{\cos \theta_{i 0}+\sqrt{\varepsilon} \cos \theta_{t 0}},
$$

while Fresnel transmission coefficient (for the transmission at the "lens" output surface) is

$$
T_{\|}=\frac{2 \cos \theta_{i}}{\cos \theta_{i}+\sqrt{\varepsilon} \cos \theta_{t}} .
$$

"Orthogonal" polarization $(\perp)$ contains components $H_{z \omega}$, $H_{\rho \omega}$, and $E_{\varphi \omega}$. Corresponding Fresnel coefficients are

$$
R_{\perp}=\frac{\sqrt{\varepsilon} \cos \theta_{i 0}-\cos \theta_{t 0}}{\sqrt{\varepsilon} \cos \theta_{i 0}+\cos \theta_{t 0}},
$$

$$
T_{\perp}=\frac{2 \sqrt{\varepsilon} \cos \theta_{i}}{\sqrt{\varepsilon} \cos \theta_{i}+\cos \theta_{t}} .
$$

The angle of incidence $\theta_{i}$ can be obtained out of Snell's law, $\sqrt{\varepsilon} \sin \theta_{i}=\sin \theta_{t}$, while $\theta_{t}$ is calculated a bit later.

It should be emphasized here that for $\beta=\beta_{0}$ a CR wave with $\|$ polarization before the lens contains only components $E_{\rho \omega}$ and $H_{\varphi \omega}$ (see, for example, Eq. (22) from [28] where $\theta_{i 2}$ corresponds to $\theta_{i}^{\prime}$ from this paper), i.e., electric field is radially polarized, while $\mathrm{CR}$ wave with $\perp$ polarization contains only components $H_{\rho \omega}$ and $E_{\varphi \omega}$, i.e., magnetic field is radially polarized.

Based on the above considerations, we can write out the transmitted field at the outer surface of the aperture which is determined via the component of the field orthogonal to the plane of incidence, i.e., $H_{\varphi \omega}$ for $\|$-polarization and $E_{\varphi \omega}$ for $\perp$-polarization. First, at the inner side of the aperture we have (see $[25,26,28]$ for details):

$$
\begin{aligned}
H_{\varphi \omega}^{a-}= & \frac{q \omega e^{i k_{0} \sqrt{\varepsilon}\left(z_{0} \cos \theta_{i}^{\prime}+\rho_{0} \sin \theta_{i}^{\prime}\right)-\frac{3 \pi i}{4}}}{i \pi v^{2} \gamma^{2}} \sqrt{\frac{2}{\pi \rho_{0} s}} \\
& \times \frac{i k_{0}}{s^{2}}\left\{-\varepsilon s I_{0}\left(r_{0} \sigma_{0}\right) \tilde{A}_{0}^{(E 2)}+2 \sum_{\nu=1}^{\infty} I_{\nu}\left(r_{0} \sigma_{0}\right) e^{\frac{i \pi(1-\nu)}{2}}\right. \\
& \left.\times \cos (\nu \varphi)\left[\varepsilon \tilde{A}_{\nu}^{(E 2)}\left(i s-\frac{1}{2 \rho_{0}}\right)-\frac{i \nu}{\beta \rho_{0}} \tilde{A}_{\nu}^{(H 2)}\right]\right\},
\end{aligned}
$$

$$
\begin{aligned}
E_{\varphi \omega}^{a-}= & \frac{q \omega e^{i k_{0} \sqrt{\varepsilon}\left(z_{0} \cos \theta_{i}^{\prime}+\rho_{0} \sin \theta_{i}^{\prime}\right)-\frac{3 \pi i}{4}}}{i \pi v^{2} \gamma^{2}} \sqrt{\frac{2}{\pi \rho_{0} s}} \\
& \times \frac{i k_{0}}{s^{2}} 2 \sum_{\nu=1}^{\infty} I_{\nu}\left(r_{0} \sigma_{0}\right) e^{\frac{i \pi(1-\nu)}{2}} \\
& \times \sin (\nu \varphi)\left[i \tilde{A}_{\nu}^{(H 2)}\left(i s-\frac{1}{2 \rho_{0}}\right)-\frac{\nu}{\beta \rho_{0}} \tilde{A}_{\nu}^{(E 2)}\right], \\
\tilde{A}_{\nu}^{(E 2)}= & \tilde{A}_{\nu}^{(E 1)} \frac{I_{\nu}}{H_{\nu}}+\frac{K_{\nu}}{H_{\nu}}, \quad \tilde{A}_{\nu}^{(H 2)}=\tilde{A}_{\nu}^{(H 1)} \frac{I_{\nu}}{H_{\nu}}, \\
\tilde{A}_{\nu}^{(E 1)}= & \frac{1}{\Delta_{\nu} H_{\nu}^{2}}\left\{-\left[\nu(\beta a)^{-1} I_{\nu}\left(\sigma_{0}^{2}+s^{2}\right)\right]^{2} H_{\nu}^{2} K_{\nu} I_{\nu}^{-1}\right. \\
& \left.+\left[\sigma_{0}^{2} s H_{\nu}^{\prime} I_{\nu}+s^{2} \sigma_{0} I_{\nu}^{\prime} H_{\nu}\right]\left[\sigma_{0}^{2} s \varepsilon H_{\nu}^{\prime} K_{\nu}+s^{2} \sigma_{0} K_{\nu}^{\prime} H_{\nu}\right]\right\}
\end{aligned}
$$



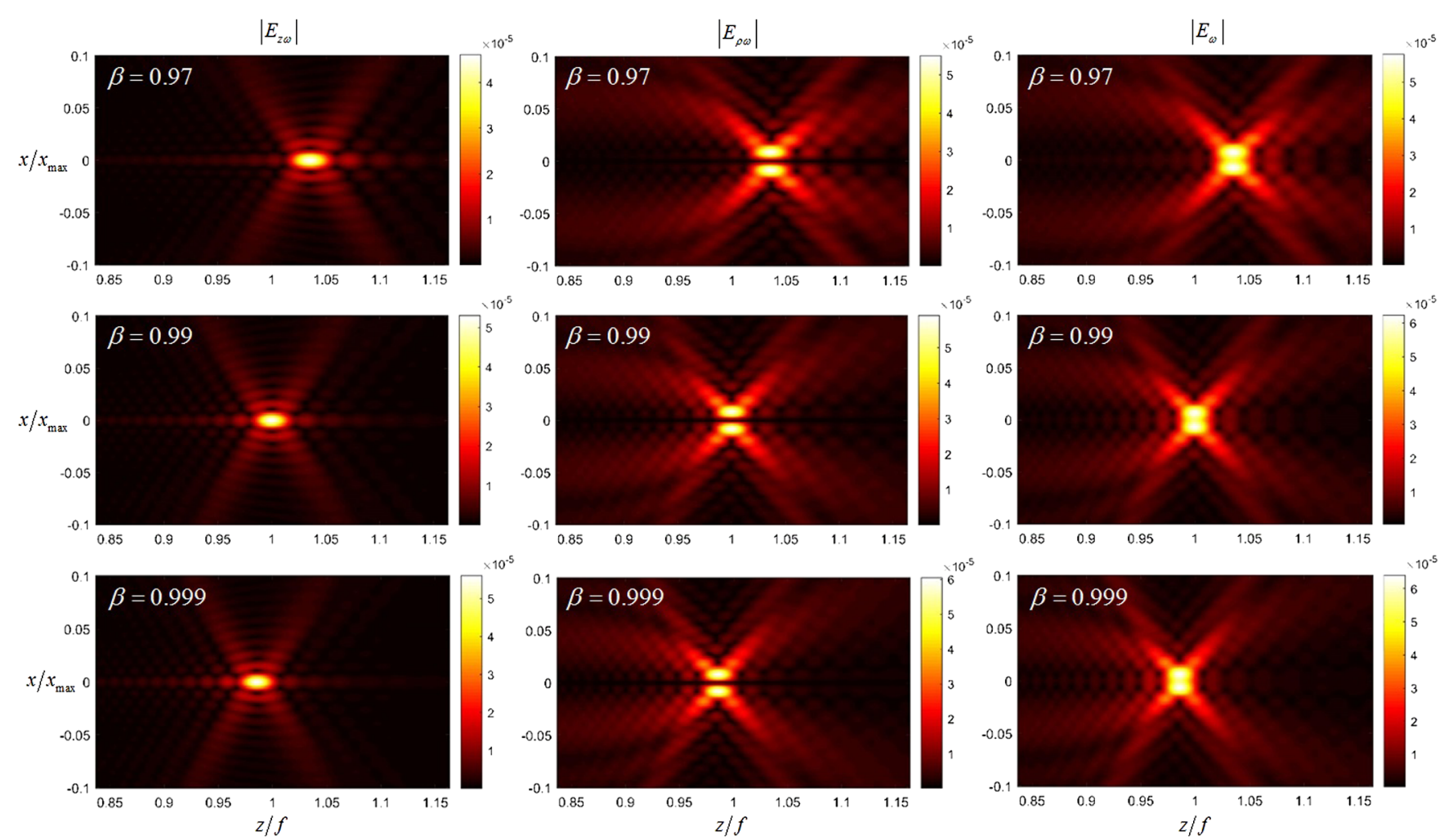

FIG. 2. Field distribution (electric field per unit frequency) over $z x$-plane ( $x$ is normalized by $x_{\max }, z$ is normalized by $f$ ) for symmetrical case $r_{0}=0$ and various charge velocities $\beta$ : top row for $\beta=0.97$, middle row for $\beta=0.99$, bottom row for $\beta=0.999$. Concentrator has been designed for $\varepsilon=4$ and $\beta_{0}=0.99$ with the following parameters: $x_{\max }=f=23.9 \mathrm{~cm}, a=c / \omega=0.047 \mathrm{~cm}$, $\alpha=30^{\circ}, \omega=2 \pi \cdot 100 \mathrm{GHz}$. Field units are $\mathrm{Vms}^{-1}, q=1 \mathrm{nC}$.

$$
\begin{aligned}
\tilde{A}_{\nu}^{(H 1)}= & \frac{\nu I_{\nu}\left(\sigma_{0}^{2}+s^{2}\right)}{i \beta a \Delta_{\nu} H_{\nu}}\left\{\left[\sigma_{0}^{2} s \varepsilon H_{\nu}^{\prime} K_{\nu}+s^{2} \sigma_{0} K_{\nu}^{\prime} H_{\nu}\right]\right. \\
& \left.-K_{\nu} I_{\nu}^{-1}\left[\sigma_{0}^{2} s \varepsilon H_{\nu}^{\prime} I_{\nu}+s^{2} \sigma_{0} I_{\nu}^{\prime} H_{\nu}\right]\right\},
\end{aligned}
$$

$$
\begin{gathered}
\Delta_{\nu}=\left[\nu(\beta a)^{-1} I_{\nu}\left(\sigma_{0}^{2}+s^{2}\right)\right]^{2} \\
-\frac{\left[\sigma_{0}^{2} s \varepsilon H_{\nu}^{\prime} I_{\nu}+s^{2} \sigma_{0} I_{\nu}^{\prime} H_{\nu}\right]\left[\sigma_{0}^{2} s H_{\nu}^{\prime} I_{\nu}+s^{2} \sigma_{0} I_{\nu}^{\prime} H_{\nu}\right]}{H_{\nu}^{2}}, \\
I_{\nu} \equiv I_{\nu}\left(a \sigma_{0}\right), \quad H_{\nu} \equiv H_{\nu}^{(1)}(a s), \\
\left.I_{\nu}^{\prime} \equiv \frac{d I_{\nu}(\xi)}{d \xi}\right|_{\xi=a \sigma_{0}},\left.\quad H_{\nu}^{\prime} \equiv \frac{d H_{\nu}^{(1)}(\xi)}{d \xi}\right|_{\xi=a s}, \\
K_{\nu} \equiv K_{\nu}\left(a \sigma_{0}\right),\left.\quad K_{\nu}^{\prime} \equiv \frac{d K_{\nu}(\xi)}{d \xi}\right|_{\xi=a \sigma_{0}},
\end{gathered}
$$

where $\sigma_{0}=k_{0} \sqrt{1-\beta^{2}}, s=k_{0} / \beta \sqrt{\varepsilon \beta^{2}-1}, \gamma$ is a Lorentz factor, $H_{\nu}^{(1)}$ is a Hankel function, $I_{\nu}$ and $K_{\nu}$ are modified Bessel and Hankel functions, correspondingly. The transmitted fields at the outer surface of the aperture are

$$
\begin{array}{ll}
H_{\varphi \omega}^{a}=T_{\|} H_{\varphi \omega}^{a-}, & \vec{E}_{\omega}^{\|}=H_{\varphi \omega}^{a}\left[\vec{e}_{\varphi}, \vec{e}_{k}\right], \\
E_{\varphi \omega}^{a}=T_{\perp} E_{\varphi \omega}^{a-}, & \vec{H}_{\omega}^{\perp}=E_{\varphi \omega}^{a}\left[\vec{e}_{k}, \vec{e}_{\varphi}\right],
\end{array}
$$

therefore

$$
\begin{gathered}
E_{\rho \omega}^{a}=H_{\varphi \omega}^{a} e_{k z}, \quad E_{z \omega}^{a}=-H_{\varphi \omega}^{a} e_{k \rho}, \\
H_{\rho \omega}^{a}=-E_{\varphi \omega}^{a} e_{k z}, \quad H_{z \omega}^{a}=E_{\varphi \omega}^{a} e_{k \rho} .
\end{gathered}
$$

Here the unit vector of the transmitted wave $\vec{e}_{k}$ is the following:

$$
\begin{aligned}
& e_{k \rho}=n_{\rho} \cos \theta_{t}-n_{z} \sin \theta_{t}, \\
& e_{k z}=n_{\rho} \sin \theta_{t}+n_{z} \cos \theta_{t} .
\end{aligned}
$$

The last point is the refraction angle $\theta_{t}$ which can be calculated using the phase term from (14), (15) and metric tensor $g$ of the outer surface (2) (see [38,40] for details):

$$
\begin{aligned}
\sin \theta_{t}= & \frac{\sqrt{\varepsilon}}{\sqrt{1-2 \sqrt{\varepsilon} \cos u+\varepsilon}} \\
& \times\left[\sin \left(2 \alpha-\theta_{p}+u\right)-\sqrt{\varepsilon} \sin \left(2 \alpha-\theta_{p}\right)\right] .
\end{aligned}
$$



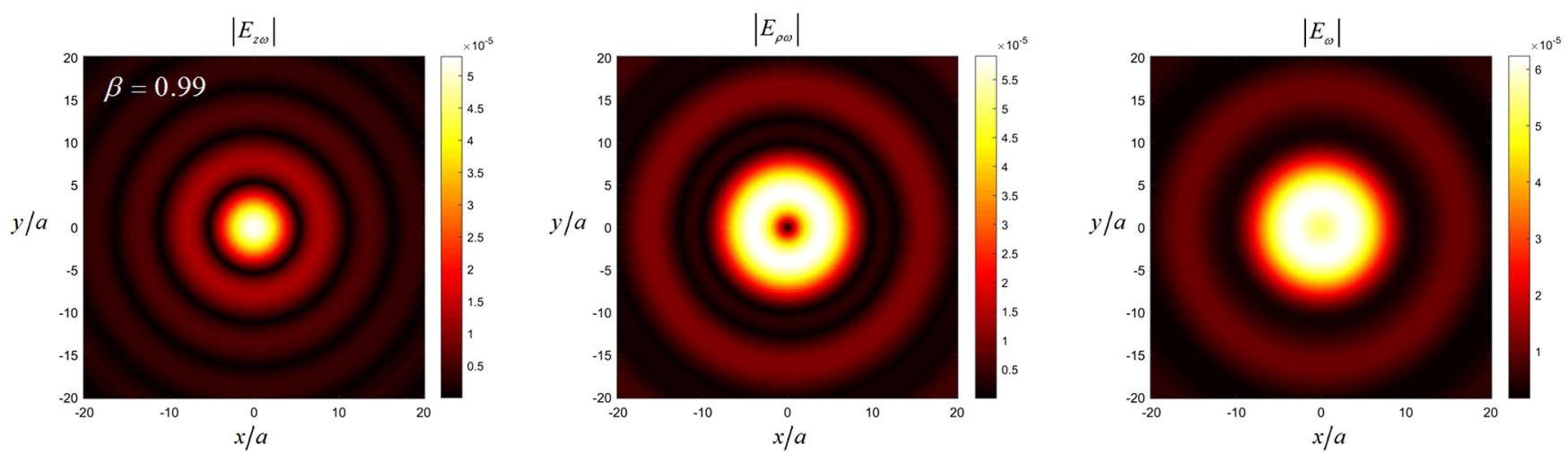

FIG. 3. Two-dimensional field distribution (electric field per unit frequency) over $x y$-plane ( $x$ and $y$ are normalized by channel radius a) for symmetrical case $r_{0}=0, \beta=0.99$ and $z=z_{f}$ (focal plane). Other parameters are the same as in Fig 2.

Now all the things needed for the evaluation of the integral (4) are ready.

\section{NUMERICAL RESULTS}

Here we present results of EM field calculation in the area outside the target (mainly, near the focal point which is of the most interest) using Eq. (4). The limits of integration over $\varphi$ are $(0,2 \pi)$, while the limits of integration over $u$ $\left(u_{\min }\right.$ and $\left.u_{\max }\right)$ are determined by transverse dimensions of the concentrator. A numerical code was realized in MATLAB with the use of Parallel Computing Toolbox for evaluation of integrals (4).

Below we present results for a point charge $q$ with a charge and current density having the form of Eq. (B2) from [25] where dependence on $z$ and $t$ is $\delta(z-v t), \delta$ is a Dirac delta function. These results can be easily generalized for a thin bunch with arbitrary longitudinal charge density distribution $\eta(z-v t)$. Since we calculate EM field in the frequency domain, to obtain formulas related to the case of such a bunch one should substitute $q \rightarrow 2 \pi q \tilde{\eta}(\omega / v)$, where $\tilde{\eta}(\omega / v)$ is the Fourier transform

$$
\tilde{\eta}(\omega / v)=(2 \pi)^{-1} \int_{-\infty}^{+\infty} \eta(\zeta) e^{-i \zeta \omega / v} d \zeta
$$

For example, in the case of Gaussian bunch with the rms half-length $\lambda_{G}$,

$$
\eta_{\mathrm{G}}(z-v t)=\frac{1}{\sqrt{2 \pi} \lambda_{G}} \exp \left(\frac{-(z-v t)^{2}}{2 \lambda_{G}^{2}}\right),
$$

one should substitute

$$
q \rightarrow q \exp \left(-\frac{\omega^{2}}{\omega_{G}^{2}}\right), \quad \omega_{G}=\frac{\sqrt{2} v}{\lambda_{G}} .
$$

Equation (IV) means that only frequencies $\omega \lesssim \omega_{G}$ (or wavelengths $\lambda \gtrsim \lambda_{G}$ ) contribute coherently to EM field.
Figure 2 illustrates distribution of absolute values of $E_{z \omega}$, $E_{\rho \omega}$ and total field $E_{\omega}$ over $z x$-plane for $r_{0}=0$. One can see that transverse field $E_{\rho \omega}$ is negligible near $z$-axis, which is natural due to the symmetry considerations, while longitudinal field $E_{z \omega}$ is dominant there. It is worth noting that maximum values of transverse and longitudinal fields are practically equal. This is more favorable compared to the "single-refraction" concentrator [25]. As one can see, the position of the focal spot changes with the change in $\beta$ but for $\beta=0.99$ (the velocity which concentrator is designed for) the spot is exactly for $z=z_{f}$ which is natural. Figure 3 illustrates similar distribution over $x y$-plane. One can conclude that this distribution is very close to the case of single-refraction concentrator.

The field distribution for the case with a charge shifted from the $z$-axis $\left(\varphi_{0}=0, r_{0} \neq 0\right)$ is shown in Fig. 4 for the focal plane $z=z_{f}$. For calculations, two asymmetric modes (which are generated in the asymmetric case) were taken into account.

It should be underlined that in this paper we are mostly considering concentrators for relativistic charges, $\beta \rightarrow 1$, which are most interesting for particle accelerator applications and which can be effectively used with this device. As it was discussed in [25], EM field of asymmetric modes is proportional to the term $I_{\nu}\left(r_{0} \sigma_{0}\right), \nu>1$, while $\sigma_{0} \sim \sqrt{1-\beta^{2}}$. Therefore asymmetry in the EM field produced by both concentrators (single-refraction and axicon-based) will be essentially weaker for relativistic particles compared to nonrelativistic ones. However, since the single-refraction concentrator is only convenient for relatively slow particles, asymmetry issues are essential for its operation. On the contrary, an axicon-based concentrator is most suitable for relativistic charges and the shift of the trajectory will affect it weaker. To illustrate this, we chose a moderate relativistic velocity $\beta=0.9$ and consider large offsets. As one can see from Fig. 4, even if the charge trajectory is close to the channel wall only small asymmetry in the longitudinal field can be observed: the field maximum is slightly shifted to positive $x$. A small redistribution 

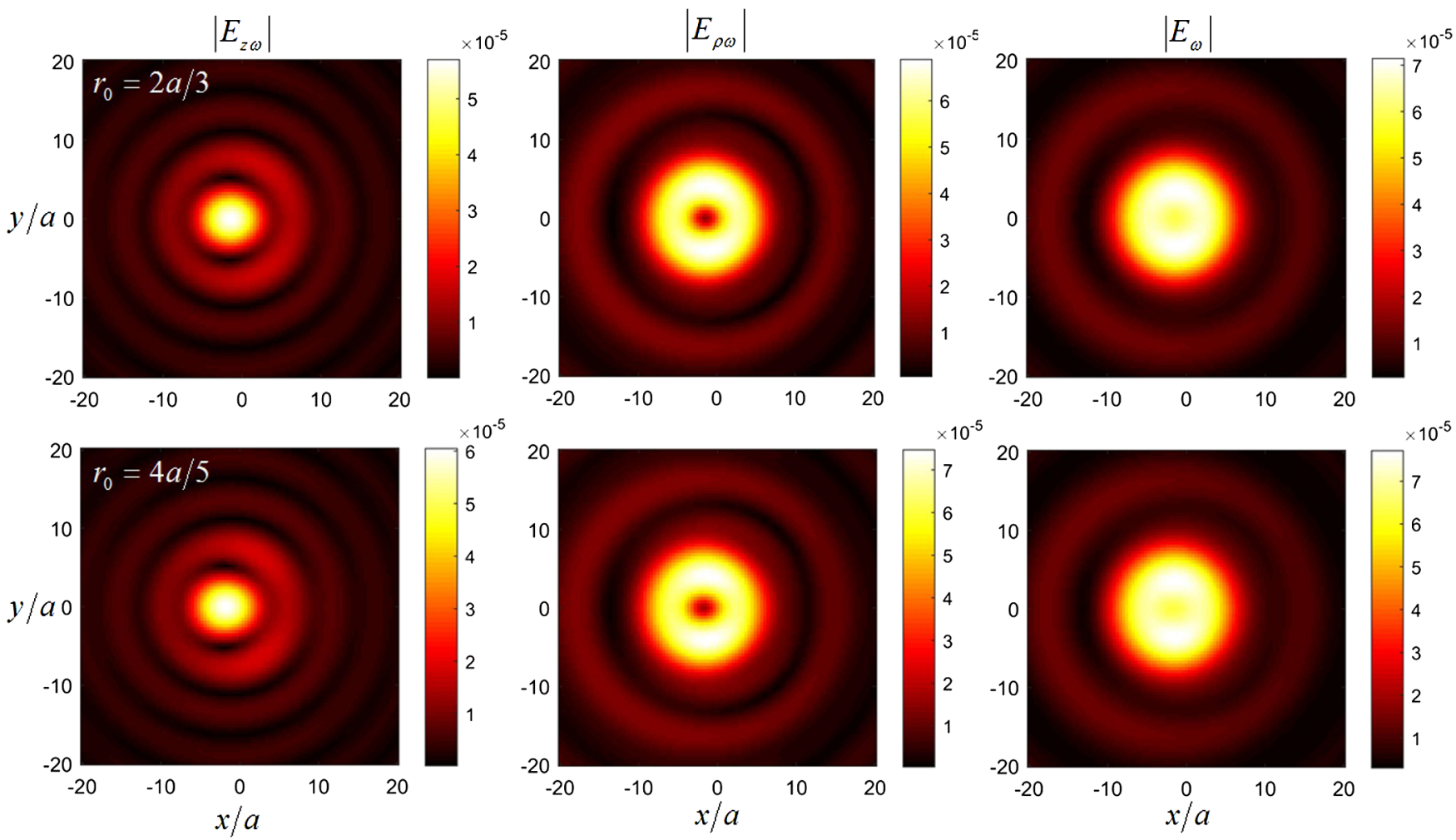

FIG. 4. Two-dimensional field distribution (electric field per unit frequency) over $x y$-plane ( $x$ and $y$ are normalized by channel radius $a$ ) for two asymmetric cases $r_{0}=3 a / 4$ and $r_{0}=4 a / 5, \varphi_{0}=0, \beta=0.9$ and $z=z_{f}$ (focal plane). Other parameters are the same as in Fig 2.

of the transverse field in $y$-direction can be also observed. Again, magnitudes of transverse and longitudinal fields are of the same order.

\section{CONCLUSION}

In the present paper, we have presented the analytical and numerical investigation of the EM radiation produced by a point charge moving through the new type of dielectric concentrator for Cherenkov radiation-axicon-based concentrator for CR. The first advantage of this target compared to the "ordinary" one (single-refraction concentrator, see $[23,25,26])$ is the possibility to choose an appropriate cone angle to realize concentration of $\mathrm{CR}$ produced by charged particles with arbitrary large velocity including relativistic bunches which are of most practical importance. Another positive distinction is that maximum longitudinal electric field is of the same order as the maximum transverse field which was not the case for the ordinary device (the longitudinal field was typically lower in maximum). The next feature is that generation of asymmetric modes (which disturb the symmetric field distribution in the focal plane) can be much less important for this target even for extremely large offsets of the bunch trajectory, which is especially the case for ultrarelativistic bunches. Overall, the discussed concentrator can be of essential importance for various applications with relativistic charged particle bunches from modern accelerators which are mainly relativistic. For example, a high-power wide-band longitudinally polarized CR can be obtained with this device, both in visible and terahertz regions. As it has been shown, the CR field inside the hollow axicon possesses radial polarization (after the first reflection from the cone generatrix) resulting in the strong longitudinal electric field near the rotation axis inside the focal spot, while the transverse electric field is close to zero here. This longitudinally polarized light is attractive for various applications, for example, it can be potentially used for bunch modulation and particle acceleration.

\section{ACKNOWLEDGMENTS}

This work was supported by the Russian Science Foundation, Grant No. 18-72-10137.

[1] P. A. Čerenkov, Visible radiation produced by electrons moving in a medium with velocities exceeding that of light, Phys. Rev. 52, 378 (1937).

[2] I. E. Tamm and I. M. Frank, Coherent radiation of fast electron in medium, Compt. Rend. Acad. Sci. URSS 14, 109 (1937). 
[3] J. V. Jelley, Čerenkov Radiation and its Applications (Pergamon, Oxford, 1958).

[4] V. P. Zrelov, Vavilov-Cherenkov Radiation in High-Energy Physics (Israel Program for Scientific Translations, Jerusalem, 1970).

[5] B. M. Bolotovskii, Theory of Cerenkov radiation (III), Sov. Phys. Usp. 4, 781 (1962).

[6] S. Antipov, C. Jing, A. Kanareykin, J. E. Butler, V. Yakimenko, M. Fedurin, K. Kusche, and W. Gai, Experimental demonstration of wakefield effects in a THz planar diamond accelerating structure, Appl. Phys. Lett. 100, 132910 (2012).

[7] C. Jing, S. Antipov, M. Conde, W. Gai, G. Ha, W. Liu, N. Neveu, J. Power, J. Qiu, J. Shi, D. Wang, and E. Wisniewski, Electron acceleration through two successive electron beam driven wakefield acceleration stages, Nucl. Instrum. Methods Phys. Res., Sect. A 898, 72 (2018).

[8] B. D. O'Shea, G. Andonian, S. Barber, K. Fitzmorris, S. Hakimi, J. Harrison, P. D. Hoang, M. J. Hogan, B. Naranjo, O. B. Williams, V. Yakimenko, and J. Rosenzweig, Observation of acceleration and deceleration in gigaelectronvolt-per-metre gradient dielectric wakefield accelerators, Nat. Commun. 7, 12763 (2016).

[9] A. P. Potylitsyn, S. Y. Gogolev, D. V. Karlovets, G. A. Naumenko, Y. A. Popov, M. V. Shevelev, and L. G. Sukhikh, Coherent Cherenkov radiation from a short bunch passing near a target and possibility of a bunch length diagnostics, in Proceedings of the International Particle Accelerator Conference, Kyoto, Japan (ICR, Kyoto, 2010), pp. 1074-1076.

[10] R. Kieffer, L. Bartnik, M. Bergamaschi, V. V. Bleko, M. Billing, L. Bobb, J. Conway, M. Forster, P. Karataev, A. S. Konkov, R. O. Jones, T. Lefevre, J. S. Markova, S. Mazzoni, Y. P. Fuentes, A. P. Potylitsyn, J. Shanks, and S. Wang, Direct Observation of Incoherent Cherenkov Diffraction Radiation in the Visible Range, Phys. Rev. Lett. 121, 054802 (2018).

[11] A. Curcio, M. Bergamaschi, R. Corsini, W. Farabolini, D. Gamba, L. Garolfi, R. Kieffer, T. Lefevre, S. Mazzoni, K. Fedorov, J. Gardelle, A. Gilardi, P. Karataev, K. Lekomtsev, T. Pacey, Y. Saveliev, A. Potylitsyn, and E. Senes, Noninvasive bunch length measurements exploiting Cherenkov diffraction radiation, Phys. Rev. Accel. Beams 23, 022802 (2020).

[12] T. Takahashi, Y. Shibata, K. Ishi, M. Ikezawa, M. Oyamada, and Y. Kondo, Observation of coherent Čerenkov radiation from a solid dielectric with short bunches of electrons, Phys. Rev. E 62, 8606 (2000).

[13] S. Antipov, M. Babzien, C. Jing, M. Fedurin, W. Gai, A. Kanareykin, K. Kusche, V. Yakimenko, and A. Zholents, Subpicosecond Bunch Train Production for a Tunable mJ Level THz Source, Phys. Rev. Lett. 111, 134802 (2013).

[14] S. N. Galyamin, A. V. Tyukhtin, S. Antipov, and S. S. Baturin, Terahertz radiation from an ultra-relativistic charge exiting the open end of a waveguide with a dielectric layer, Opt. Express 22, 8902 (2014).

[15] N. Sei, T. Sakai, K. Hayakawa, T. Tanaka, Y. Hayakawa, K. Nakao, K. Nogami, and M. Inagaki, Proposal of coherent Cherenkov radiation matched to circular plane wave for intense terahertz light source, Phys. Lett. A 379, 2399 (2015).

[16] N. Sei and T. Takahashi, First demonstration of coherent Cherenkov radiation matched to circular plane wave, Sci. Rep. 7, 17440 (2017).

[17] D. Wang, X. Su, L. Yan, Y. Du, Q. Tian, Y. Liang, L. Niu, W. Huang, W. Gai, C. Tang, and S. Antipov, Phase control with two-beam interferometry method in a terahertz dielectric wakefield accelerator, Appl. Phys. Lett. 111, 174102 (2017).

[18] D. Wang, X. Su, Y. Du, Q. Tian, Y. Liang, L. Niu, W. Huang, W. Gai, L. Yan, C. Tang, and S. Antipov, Nonperturbing $\mathrm{THz}$ generation at the Tsinghua university accelerator laboratory $31 \mathrm{MeV}$ electron beamline, Rev. Sci. Instrum. 89, 093301 (2018).

[19] M. Bass, P. A. Franken, J. F. Ward, and G. Weinreich, Optical Rectification, Phys. Rev. Lett. 9, 446 (1962).

[20] J. Hebling, K.-L. Yeh, M. C. Hoffmann, B. Bartal, and K. A. Nelson, Generation of high-power terahertz pulses by tilted-pulse-front excitation and their application possibilities, J. Opt. Soc. Am. B 25, B6 (2008).

[21] M. I. Bakunov, R. V. Mikhaylovskiy, S. B. Bodrov, and B.S. Luk'yanchuk, Reversed Cherenkov emission of terahertz waves from an ultrashort laser pulse in a sandwich structure with nonlinear core and left-handed cladding, Opt. Express 18, 1684 (2010).

[22] M. I. Bakunov, E. S. Efimenko, S. D. Gorelov, N. A. Abramovsky, and S. B. Bodrov, Efficient Cherenkov-type optical-to-terahertz converter with terahertz beam combining, Opt. Lett. 45, 3533 (2020).

[23] S. N. Galyamin and A. V. Tyukhtin, Dielectric Concentrator for Cherenkov Radiation, Phys. Rev. Lett. 113, 064802 (2014).

[24] A. V. Tyukhtin, V. V. Vorobev, S. N. Galyamin, and E. S. Belonogaya, Radiation of a charge moving along the boundary of dielectric prism, Phys. Rev. Accel. Beams 22, 012802 (2019).

[25] S. N. Galyamin, V. V. Vorobev, and A. V. Tyukhtin, Radiation of a charge in dielectric concentrator for Cherenkov radiation: Off-axis charge motion, Phys. Rev. Accel. Beams 22, 083001 (2019).

[26] S. N. Galyamin, V. V. Vorobev, and A. V. Tyukhtin, Erratum: Radiation of a charge in dielectric concentrator for Cherenkov radiation: Off-axis charge motion, Phys. Rev. Accel. Beams 22, 109901 (2019).

[27] S. Galyamin, A. Tyukhtin, and V. Vorobev, Focusing the Cherenkov radiation using dielectric concentrator: Simulations and comparison with theory, J. Instrum. 13, C02029 (2018).

[28] A. V. Tyukhtin, S. N. Galyamin, V. V. Vorobev, and A. A. Grigoreva, Cherenkov radiation of a charge flying through the "inverted" conical target, arXiv:2005.00759.

[29] S. N. Khonina and S. A. Degtyarev, Analysis of the formation of a longitudinally polarized optical needle by a lens and axicon under tightly focused conditions, J. Opt. Technol. 83, 197 (2016).

[30] A.-P. Yu, G. Chen, Z.-H. Zhang, Z.-Q. Wen, L.-R. Dai, K. Zhang, S.-L. Jiang, Z.-X. Wu, Y.-Y. Li, C.-T. Wang, and X.-G. Luo, Creation of sub-diffraction longitudinally 
polarized spot by focusing radially polarized light with binary phase lens, Sci. Rep. 6, 38859 (2016).

[31] L. Turquet, X. Zang, J.-P. Kakko, H. Lipsanen, G. Bautista, and M. Kauranen, Demonstration of longitudinally polarized optical needles, Opt. Express 26, 27572 (2018).

[32] D. N. Gupta, N. Kant, D. E. Kim, and H. Suk, Electron acceleration to $\mathrm{GeV}$ energy by a radially polarized laser, Phys. Lett. A 368, 402 (2007).

[33] Y. I. Salamin, Low-diffraction direct particle acceleration by a radially polarized laser beam, Phys. Lett. A 374, 4950 (2010).

[34] S. G. Bochkarev, K. I. Popov, and V. Y. Bychenkov, Vacuum electron acceleration by a tightly focused, radially polarized, relativistically strong laser pulse, Plasma Phys. Rep. 37, 603 (2011).
[35] J. H. McLeod, The axicon: A new type of optical element, J. Opt. Soc. Am. 44, 592 (1954).

[36] J. A. Stratton and L. J. Chu, Diffraction theory of electromagnetic waves, Phys. Rev. 56, 99 (1939).

[37] A.Z. Fradin, Microwave Antennas (Pergamon, Oxford, 1961).

[38] S. N. Galyamin and A. V. Tyukhtin, Dielectric concentrator for Cherenkov radiation: Sensitivity and field near the focus, Nucl. Instrum. Methods Phys. Res., Sect. B 402, 185 (2017).

[39] A. V. Tyukhtin, S. N. Galyamin, and V. V. Vorobev, Peculiarities of Cherenkov radiation from a charge moving through a dielectric cone, Phys. Rev. A 99, 023810 (2019).

[40] V. A. Fok, Electromagnetic Diffraction and Propagation Problems (Pergamon Press, Oxford, 1965). 